\title{
Melting and Crystallization of Ultra-High Molecular Weight Polyethylene with Appearance of Hexagonal Phase I. Melting Processes of Fibers under Constrained State
}

\author{
Shinsuke Tsubakihara, Atsushi NaKamura, and Munehisa Yasuniwa \\ Department of Applied Physics, Faculty of Science, Fukuoka University, \\ Jonan-ku, Fukuoka 814-80, Japan
}

(Received September 8, 1995)

\begin{abstract}
Melting behavior under constrained state was studied for two kinds of commercial ultra-high molecular weight polyethylene (PE) fibers with different molecular weights by means of differential scanning calorimetry (DSC) and $\mathrm{X}$-ray measurement. DSC melting curves of constrained samples were compared with those of unconstrained ones. It was shown that the constrained sample mainly melts through two endothermic peaks 2 and 3 , while the unconstrained sample mainly melts through single endothermic peak 1 whose peak temperature is lower than those of peaks 2 and 3 . These peak temperatures were very reproducible for each sample. Peak 1 of the unconstrained sample was attributed to the melting of orthorhombic phase composed of so-called extended-chain crystals. On the other hand, based on the X-ray data, peaks 2 and 3 of the constrained sample were assigned to a transition orthorhombic to hexagonal phase and a melting of the hexagonal phase, respectively. Based on the visual observation, peak 3 was also shown to be accompanied by the break and rapid thermal-contraction of the sample. These peculiar transition and melting mechanisms of PE under the constrained state were discussed on the basis of a free energy diagram that can explain the experimental results successfully.

KEY WORDS Polyethylene / Ultra-High Molecular Weight / Fiber / Melting / Constrained State /

Hexagonal / Phase Transition / Free Energy Diagram /
\end{abstract}

Two decades ago, ultra-drawn polyethylene (PE) fiber made from ultra-high molecular weight PE (UHMW-PE) was developed, and has been commercially produced. ${ }^{1-3}$ This fiber is noted as a light and tough material because of a high tensile strength between 2 and $7 \mathrm{GPa}$ and high Young modulus above $100 \mathrm{GPa}$, which are comparable to inorganic fibers such as glass, carbon, and steel etc. However, the fiber is of poor heat resistance since a melting point of $\mathrm{PE}$ crystal is very low about $140^{\circ} \mathrm{C}$, and it has been an important subject to improve the heat resistance. Therefore, detailed studies about its melting process $^{4-8}$ and some attempts such as irradiation ${ }^{9,10}$ or chemical cross-linking ${ }^{11,12}$ have been made to find an effective method.

For such a reason, the melting process of a physically constrained PE fiber was also studied, and the constraining method was shown to raise the melting point of PE crystal by $10-15 \mathrm{~K}$, that is, improve the heatresistance slightly. ${ }^{4,7,8}$ Numerous entanglements among tie molecules in the UHMW-PE fiber play a significant role in this increase of the melting point. Moreover, it was also reported that a phase transition due to a change of crystal structure, orthorhombic to hexagonal, occurs prior to the melting. ${ }^{7,8}$ This hexagonal structure is the same as that of so-called high pressure phase of a bulk sample, which appears only at high pressures above about $300 \mathrm{MPa} .{ }^{13-15}$ In this structure, molecular chains arrange in an orderly hexagonal packing in a lateral plane perpendicular to the molecular axis, while there is no order in their direction, so that it is also termed pseudo-hexagonal structure. ${ }^{16-18}$ Such a phase transition of the constrained fiber is unique and interesting, and the phenomenon has been well known since the finding by Pennings et al. ${ }^{7}$ Further, it was also known that the phase transition gives a critical point to the heat-resistance because the strength of the fiber decreases drastically at the point. ${ }^{7}$ Accordingly, studies about the phase transition are very important, so that some studies including authors' one have been done. ${ }^{7,8,12,19}$ Nevertheless, effects of differences in type of sample and constraining method on the phase transition have not yet been examined sufficiently, and the transition mechanism is not clarified even at present. Thus, it is necessary to investigate this phenomenon more systematically and clarify the mechanism.

There is another specific effect of the numerous entanglements among molecular chains. The UHMW-PE fiber contracts considerably when it melts under an unconstrained state. This thermal-contraction is caused by the melting of included PE crystals. However, the contracted, i.e., molten sample is rubber-like and hardly flows because melt viscosity of the UHMW-PE is very high. Further, it can be elongated from the molten state at higher temperatures than its melting point. In this elongating process, it is presumed that the strain-induced crystallization, well known in a natural rubber, occurs. If this phenomenon does, mechanical properties such as the tensile strength and Young modulus are expected to recover to a certain degree. Therefore, a detailed study about the strain-induced crystallization of the contracted sample seems to extend the possibility of the PE fiber at elevated temperatures.

In addition to the application advantages, the studies about the melting of constrained sample and straininduced crystallization, which the numerous entanglements make possible, are also expected to give a lot of fundamental information with regard to melting and crystallization processes of crystalline polymers. In this work, these characteristic melting and crystallization processes of PE, which have not been studied adequately, were investigated for some kinds of fibers and highlydrawn samples made from the UHMW-PE. The results 
are reported in two papers. This paper (Part I) particularly describes the study about the melting process under the constrained state, carried out for two kinds of commercial UHMW-PE fibers by differential scanning calorimetry (DSC) and X-ray measurement. This study promotes the content that the authors already reported in a previous paper. ${ }^{19}$ At first, DSC melting curves of constrained and unconstrained samples are compared with each other, and the difference between their melting processes is shown. Next the endothermic peaks of the constrained sample are assigned based on results obtained by X-ray measurement, etc. At last, mechanisms of the transition and melting under the constrained state are discussed on the basis of a free energy diagram that is able to explain the experimental results successfully.

\section{EXPERIMENTAL}

Two kinds of commercial UHMW-PE fibers, "Spectra900" (Allied Signal Co., viscosity average molecular weight, $M_{v}=c a .2 \times 10^{6}$ ) and "Tekmilon NA310" (Mitsui Petrochemical Industries Co., $M_{v}=c a .7 \times 10^{5}$ ), were used. They were denoted by samples 1 and 2, respectively.

The melting processes of the two kinds of samples were examined under both the constrained and unconstrained states using a DSC apparatus (Perkin-Elmer DSC7). The constraining method is shown in Figure 1. The fiber samples were tightly wound round a copper ring of $4 \mathrm{~mm}$ diameter and tied at the edges, and then the excess portions were cut off. On the other hand, very short free fibers cut to a length of about $1 \mathrm{~mm}$ from the original ones were dealt with as the unconstrained samples. To obtain the adequate constraint-free samples, the original fibers had to be cut off to the short length because the longer free fiber, even if it is $5 \mathrm{~mm}$ in length, underwent considerable constraining force without being constrained by any special method. Both the constrained and unconstrained samples were then independently confined to aluminum containers of $6 \mathrm{~mm}$ diameters, and the DSC measurements were carried out at a heating rate of $5.0 \mathrm{~K} \mathrm{~min}^{-1}$. In addition, DSC curves of constrained sample- 1 were also recorded at heating rates of $0.3,0.6$, 1.2 , and $2.5 \mathrm{~K} \mathrm{~min}^{-1}$ to examine the superheating effect of the heating rate on the melting process.

Structure change of the constrained sample was examined by the X-ray measurement in the heating process. Figure 2 shows a sample holder and a heating cell made of brass. The fiber sample was fixed on the sample holder and coated with an epoxy resin for the sake of constraining it. The holder was then set in the cell as shown in the figure. A pinhole-collimated Mo- $K_{\alpha}$ beam filtered through a zirconium film was irradiated to the sample perpendicularly to the fiber axis, and its equatorial diffraction pattern was recorded with a scintillation counter. As a X-ray generator, a Rigaku Rotaflex RU-200 $(60 \mathrm{kV}$, $200 \mathrm{~mA}$ ) was used. The diffractometer was scanned over a range of $8.5^{\circ}<2 \theta<12^{\circ}$ in which main reflections of the orthorhombic and hexagonal structures were observed. Temperature of the sample was raised in steps, and the diffraction pattern was recorded at each temperature. The temperature was measured with an alumelchromel thermocouple located at a distance, $1 \mathrm{~mm}$, from the center of an X-ray exposure part.
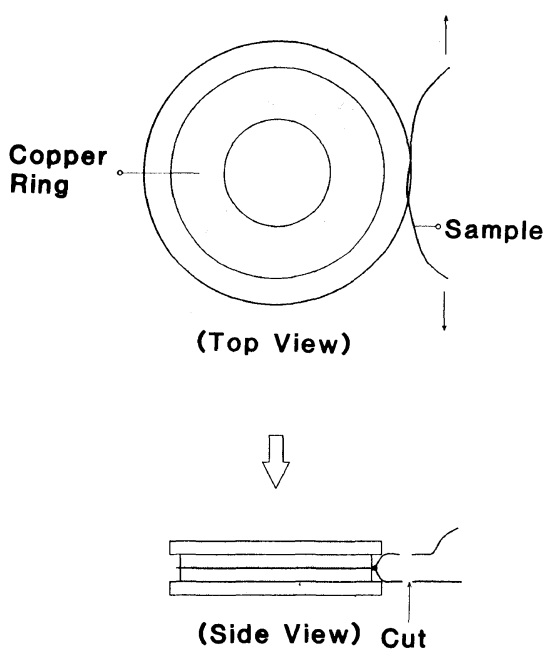

Figure 1. Constraining method of UHMW-PE fiber. The fiber sample was tightly wound round a copper ring of $4 \mathrm{~mm}$ diameter and tied at the edges, and then the excess portions were cut off. The ring was confined to an aluminum container, and DSC measurement was carried out.



Figure 2. (a) Sample holder and (b) heating cell for X-ray measurement, used to examine structure change of constrained fiber in the heating process. A fiber sample was fixed on the sample holder and coated with an epoxy resin for the sake of constraining it.

In order to examine changes of shape and length in the heating process, sample-1 constrained in the same manner as the thermal analysis was heated to two characteristic temperatures at a heating rate of $5.0 \mathrm{~K} \mathrm{~min}^{-1}$ and then quenched to room temperature at the moment when reaching each temperature. These processes were carried out with the DSC apparatus. The resulting samples were photographed, and the changes of the shape and length caused by the thermal effect were exhibited.

\section{RESULTS AND DISCUSSION}

Figure 3 shows DSC melting curves of sample-1, measured under (a) constrained and (b) unconstrained states. In the case of the constrained sample (Figure 3(a)), there are three endothermic peaks denoted as peaks $1-3$. Their peak temperatures are 143,155, and $159^{\circ} \mathrm{C}$, respectively, and peak 1 is considerably smaller than the other peaks. On the other hand, peak 1 of the unconstrained sample (Figure 3(b)), whose peak temperature is $144^{\circ} \mathrm{C}$ nearly equal to $143^{\circ} \mathrm{C}$ of the con- 


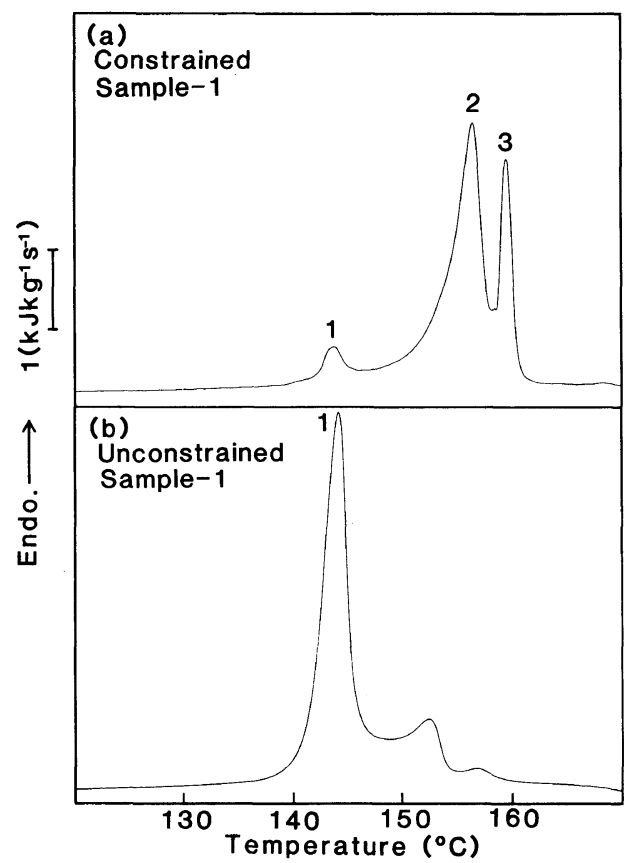

Figure 3. DSC melting curves of sample-1, measured under (a) constrained and (b) unconstrained states. The heating rate was $5.0 \mathrm{~K} \mathrm{~min}^{-1}$.

strained one, is the largest. In this case, the unconstrained sample almost melts through this endothermic peak. It is well known that the ultra-drawn PE fiber is composed of so-called extended-chain crystals. Accordingly, peak 1 is attributed to their melting under the unconstrained state. The constraining effect prevents this melting process and induces another melting one proceeding through peaks 2 and 3 instead of peak 1 . Consequently, the two peaks become predominant, while the heat quantity of peak 1 becomes very small. Probably the constraint-free portions at the tie point, which could not be cut off, are responsible for this small peak 1 .

DSC melting curves of sample-2 are shown in Figure 4. Their features are substantially the same as those of sample-1. However, the temperature interval between peaks 2 and 3 in Figure 4(a) is narrower than that in Figure 3(a), so that the two peaks are not sufficiently separated. Moreover, the right-hand shoulder peak in Figure 4(b), which is attributed to the slightly constrained portion, is smaller than that in Figure 3(b). These differences in constraining effects are probably due to the difference in molecular weights between samples-1 and -2 since the higher molecular weight sample generally undergoes more effective constraining force because of more entanglements among molecular chains.

To assign peak 2 that appears only under the constrained state, X-ray measurement of constrained samples was carried out. Figure 5 shows a temperature change of the X-ray equatorial diffraction pattern of constrained sample- 1 , measured in the heating process with the apparatus shown in Figure 2. At 153 and $154^{\circ} \mathrm{C}$, (110)o and (200)o diffraction peaks of orthorhombic structure appear, while (100)h single diffraction peak of hexagonal structure appears above $155^{\circ} \mathrm{C}$ instead of them. This indicates that the phase transition due to a change of crystal structure, orthorhombic to hexagonal, occurs at $155^{\circ} \mathrm{C}$ in the heating process. After the phase transition, the diffraction intensity of $(100) \mathrm{h}$ peak de-



Figure 4. DSC melting curves of sample-2, measured under (a) constrained and (b) unconstrained states. The heating rate was $5.0 \mathrm{~K} \mathrm{~min}^{-1}$.

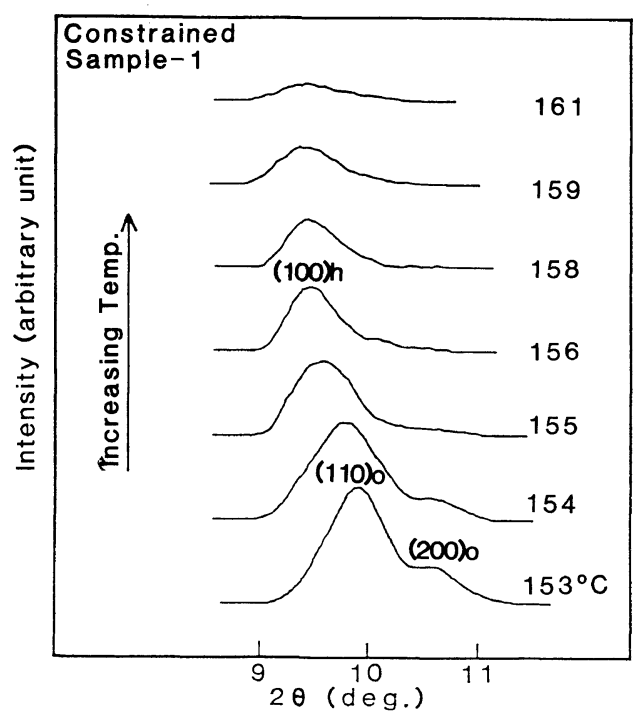

Figure 5. Temperature change of the $X$-ray equatorial diffraction pattern of constrained sample-1, measured in the heating process with the apparatus shown in Figure 2. (110)o and (200)o represent orthorhombic reflections, and $(100) \mathrm{h}$ does hexagonal one.

creases gradually with temperature and disappears eventually at $161^{\circ} \mathrm{C}$. The halo of liquid or the epoxy resin is afterward left. Figure 6 shows a result with respect to sample-2. In this case, the phase transition occurs between 152 and $153^{\circ} \mathrm{C}$. At this point, the orthorhombic and hexagonal peaks coexist. Then the constrained sample melts completely at $155^{\circ} \mathrm{C}$.

In Figure 7, the DSC melting curve of constrained sample-1 and its temperature dependence of lattice spacing $d$ calculated from the $\mathrm{X}$-ray diffraction pattern are shown on the same temperature scale. In the heating process, two orthorhombic lattice spacing curves disappear within the temperature range of peak 2 , and the hexagonal curve begins instead of them. This indicates that endothermic peak 2 of the DSC melting curve is 


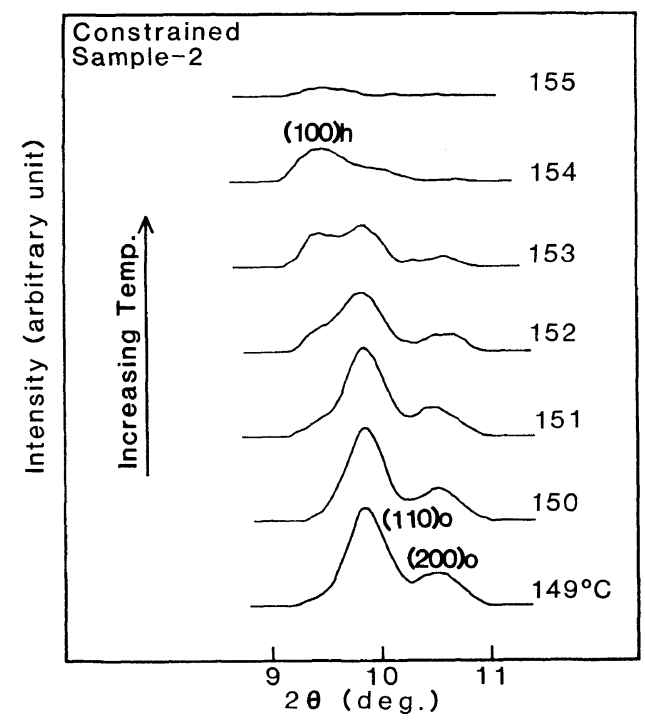

Figure 6. Temperature change of the X-ray equatorial diffraction pattern of constrained sample-2, measured in the heating process with the apparatus shown in Figure 2.

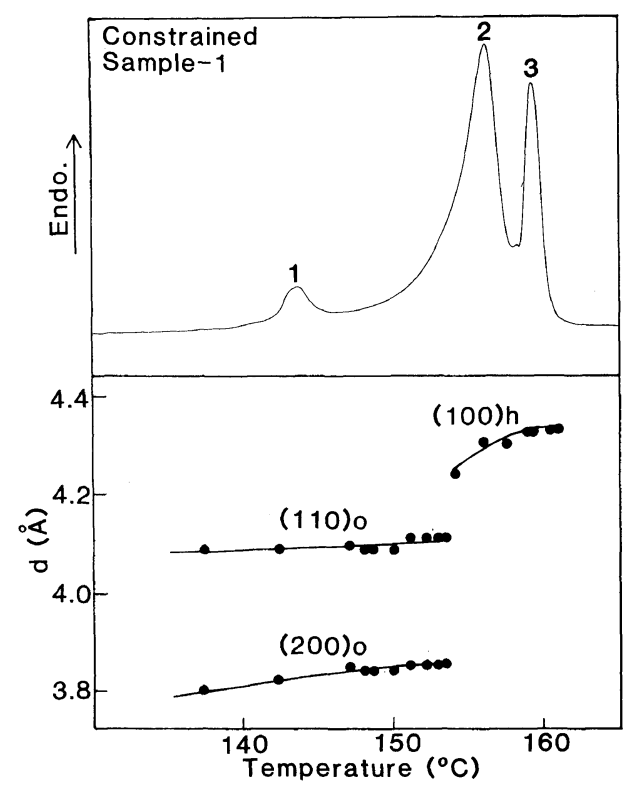

Figure 7. DSC melting curve of constrained sample-1 and its temperature change of lattice spacing $d$, obtained in the heating process.

attributed to the phase transition orthorhombic to hexagonal structure. Subsequently, the hexagonal lattice spacing increases by $c a$. $0.1 \AA$ with temperature, that is, its lattice expands appreciably, and then the curve disappears at the end of peak 3 corresponding to the complete melting. A similar result with respect to sample2 is shown in Figure 8. This result also indicates that the phase transition is responsible for peak 2. In this case, the temperature range of hexagonal is very narrow as well as the interval between peaks 2 and 3, and there is small temperature range in which two crystal structures coexist. These facts indicate that the hexagonal phase of constrained sample- 2 is not as stable as that of sample- 1 .

To examine the phenomenon occurring through peak 3 , the changes of the shape and length in the heating process were observed. Figure 9 is a photograph exhibiting their characteristic changes, taken at room tem-

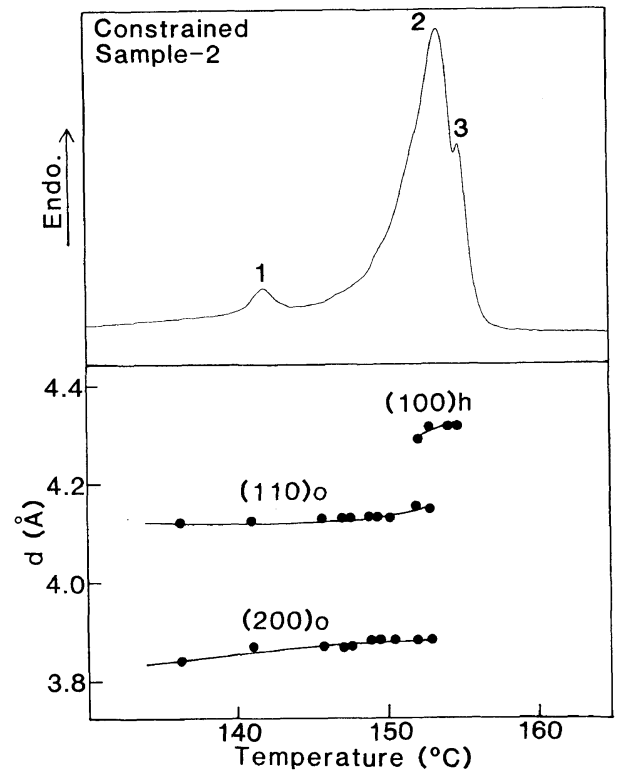

Figure 8. DSC melting curve of constrained sample-2 and its temperature change of lattice spacing $\mathrm{d}$, obtained in the heating process.

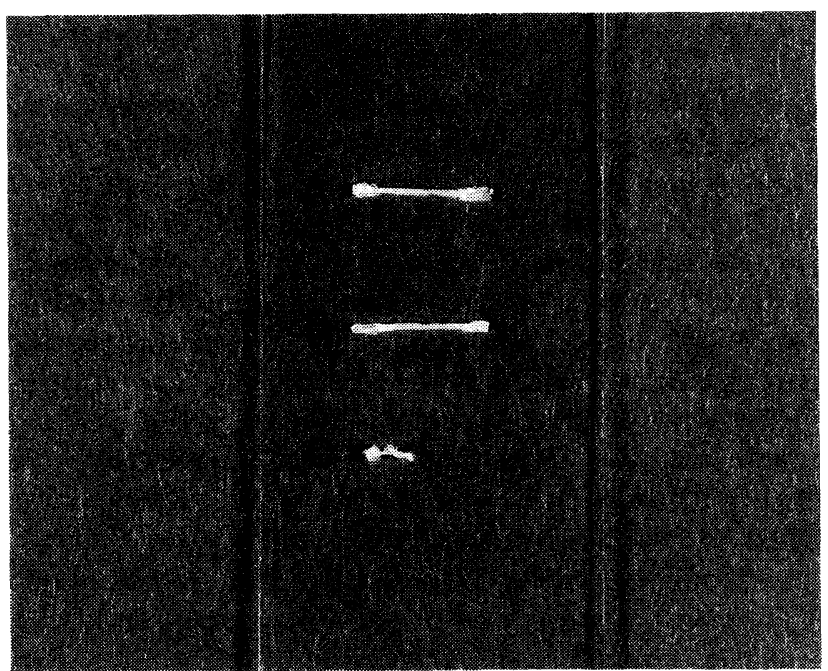

Figure 9. Photograph of sample-1, which exhibits changes of shape and length in the heating process under the constrained state. The photograph was taken at room temperature. Sample $A$ is an original sample-1, and samples B and C were obtained by annealing the same length of original one as sample $\mathrm{A}$.

perature. Sample $A$ is an original sample- 1 of a $10 \mathrm{~mm}$ length, while samples $\mathrm{B}$ and $\mathrm{C}$ were obtained by annealing the same length of original one as sample $\mathrm{A}$. The annealing conditions of samples $\mathrm{B}$ and $\mathrm{C}$ were as follows. Sample B was heated to a middle point between peaks 2 and 3 at the rate of $5 \mathrm{~K} \mathrm{~min}^{-1}$, and then quenched to room temperature at the moment when it reached the point. On the other hand, sample $\mathrm{C}$ was heated to the end point of peak 3 at the same rate, and then quenched immediately from the point. As shown in Figure 9, the shape and length of sample B are the same as those of sample A, that is, constrained sample- 1 does not change at all even after its temperature passes through peak 2 at which the phase transition occurs. On the other hand, they drastically change after it does through peak 3 . Sample $\mathrm{C}$ in the figure is very wrinkled and short. This results from a rapid thermal-contraction due to the break

Polym. J., Vol. 28, No. 6, 1996 
Table I. Peak temperatures ${ }^{a}$ of constrained sample-1

\begin{tabular}{|c|c|c|c|}
\hline \multirow{2}{*}{ No. } & Peak 1 & Peak 2 & Peak 3 \\
\hline & ${ }^{\circ} \mathrm{C}$ & ${ }^{\circ} \mathrm{C}$ & ${ }^{\circ} \mathrm{C}$ \\
\hline 1 & 143 & 156 & 159 \\
\hline 2 & 143 & 156 & 159 \\
\hline 3 & 143 & 155 & 159 \\
\hline 4 & 143 & 155 & 158 \\
\hline 5 & 143 & 154 & 158 \\
\hline Av. & 143 & 155 & 159 \\
\hline
\end{tabular}

a These data were obtained for sample-1 wound round copper ring (heating rate, $5 \mathrm{~K} \mathrm{~min}^{-1}$ ).

Table II. Peak temperatures ${ }^{\text {a }}$ of constrained sample-2

\begin{tabular}{|c|c|c|c|}
\hline \multirow{2}{*}{ No. } & Peak 1 & Peak 2 & Peak 3 \\
\hline & ${ }^{\circ} \mathrm{C}$ & ${ }^{\circ} \mathrm{C}$ & ${ }^{\circ} \mathrm{C}$ \\
\hline 1 & 142 & 154 & 155 \\
\hline 2 & 142 & 153 & 154 \\
\hline 3 & 142 & 153 & 155 \\
\hline 4 & 142 & 153 & 154 \\
\hline 5 & 142 & 153 & 155 \\
\hline Av. & 142 & 153 & 155 \\
\hline
\end{tabular}

${ }^{a}$ These data were obtained for sample-2 wound round copper ring (heating rate, $5 \mathrm{~K} \mathrm{~min}^{-1}$ ).

of the sample. From this result, it is concluded that the endothermic phenomenon at peak 3 , caused by the melting of the hexagonal phase, is accompanied by the break and rapid thermal-contraction of the sample. The same conclusion was also reached on sample- 2 .

Table I shows the peak temperatures of constrained sample-1, repeatedly measured 5 times. Each temperature of peaks $1-3$ is reproducible, that is, the transition and melting of the constrained sample occur at their individual definite temperatures. In particular, the reproducibility of peak 3 reveals that the melting of the hexagonal phase is not caused by any mechanical origin such as the break of the sample, but by a definite thermodynamic origin. If the melting is caused by the mechanical origin whose condition varies each time, the temperature of peak 3 must not be reproducible. Probably the break of the sample results from considerable decrease of the tensile strength, which is owing to the melting of the hexagonal phase. The average temperature of peak 1 related to the unconstrained residual portion is $143^{\circ} \mathrm{C}$, and those of peaks 2 and 3 related to the constrained main portion are 155 and $159^{\circ} \mathrm{C}$, respectively. The results of constrained sample- 2 are given in Table II. Each temperature in this case is also reproducible. The average temperatures of peaks 1 $\left(142^{\circ} \mathrm{C}\right)$ and $2\left(153^{\circ} \mathrm{C}\right)$ are close to those of sample-1. On the other hand, that of peak $3\left(155^{\circ} \mathrm{C}\right)$ is somewhat lower than that of sample-1. This difference implies that the melting temperature of the hexagonal phase is apt to be influenced by inherent factors of the constrained sample such as the molecular weight.

Melting curves of constrained sample-1, measured at several heating rates, $0.3,0.6,1.2,2.5$, and $5.0 \mathrm{~K} \mathrm{~min}^{-1}$, are shown in Figure 10. The peaks lower remarkably

Polym. J., Vol. 28, No. 6, 1996

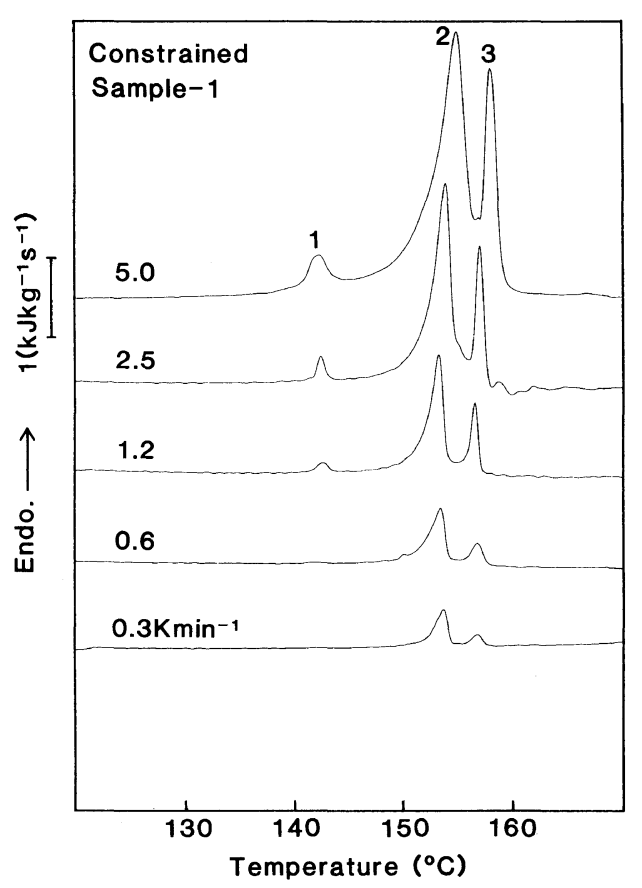

Figure 10. Melting curves of constrained sample-1, measured at several heating rates, $0.3,0.6,1.2,2.5$, and $5.0 \mathrm{~K} \mathrm{~min}^{-1}$.

with decreasing heating rate because the unit of the longitudinal axis represents heat quantity flowing into the sample per second. Except for the lowering, the melting curve profile mainly showing peaks 2 and 3 does not change essentially with the decreasing rate though peak 3 lowers relative to peak 2 in some degree. When the heating rate increases 0.3 to $5.0 \mathrm{~K} \mathrm{~min}^{-1}$, the rises of the peak temperatures, caused by the superheating effect, are less than $2^{\circ} \mathrm{C}$.

Based on the DSC and X-ray data etc., the melting behavior under the constrained state has so far been described for two kinds of PE fibers. The mechanism of this unique melting can be explained thermodynamically as follows. Figure 11 is a supposed schematic free energy diagram representing the melting process under the constrained state. For the sake of convenience, enthalpy $H$ and entropy $S$ of the fundamental thermodynamic equation

$$
G=H-T S
$$

where $G$ and $T$ are, respectively, Gibbs free energy and temperature are assumed to be constants for each phase in the considered temperature range. Consequently, the relationship between $G$ and $T$ is linear, so that $G-T$ line of each phase can be drawn. Three solid lines denoted by $\mathrm{o}, \mathrm{h}$, and $\mathrm{m}$ express orthorhombic, hexagonal, and melt phases under the constrained state, respectively. On the other hand, broken line $\mathrm{m}^{\prime}$ does a melt phase under the unconstrained state, which is the same as that the unconstrained sample takes after its melting. On account of increasing molecular motion, the hexagonal phase (h) is more disorder than the orthorhombic phase (o), and the two melt phases are further disorder. Between the constrained $(\mathrm{m})$ and unconstrained $\left(\mathrm{m}^{\prime}\right)$ melt phases, the latter is most likely to more disorder because its degree of molecular orientation is reasonably smaller than the former. Therefore, there is the following in- 


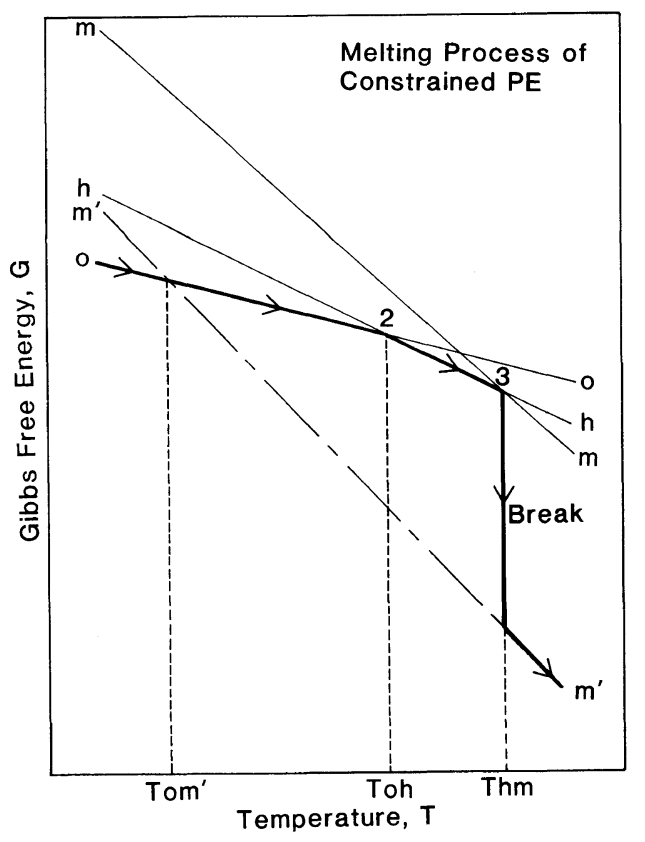

Figure 11. Schematic free energy diagram representing the melting process of the constrained UHMW-PE fiber. Three solid lines o, h, and $m$ express orthorhombic, hexagonal, and melt phases under the constrained state, respectively, while broken line $\mathrm{m}^{\prime}$ does a melt phase under the unconstrained state. In the melting process, the state of the constrained fiber proceeds along the path represented by the thick arrows.

equality among the entropies of these phases

$$
S_{\mathrm{o}}<S_{\mathrm{h}}<S_{\mathrm{m}}<S_{\mathrm{m}^{\prime}}
$$

where $S_{\mathrm{o}}$ is entropy of orthorhombic, $S_{\mathrm{h}}$ hexagonal, $S_{\mathrm{m}}$ constrained melt, and $S_{\mathrm{m}^{\prime}}$ unconstrained melt phase. Slopes of the four $G-T$ lines in the diagram, of course, increase in this order of the entropies, i.e., left to right in the inequality. In the melting process, the state of the constrained sample proceeds along the path represented by the thick arrows in Figure 11. In the first place, the sample does along orthorhombic line o. In this process, the thermal-contractile force arises in the sample itself above $T_{\text {om }^{\prime}}$, the intersection of two lines o and $\mathrm{m}^{\prime}$, because the free energy $G$ of the former is larger than that of the latter in this temperature range. This force actually arises, so that the sample contracts if the constraining force that must be equal to the contractile one disappears or is eliminated. As shown in the figure, the constrained sample then transfers to hexagonal line $\mathrm{h}$ at $T_{\mathrm{oh}}$, the intersection of lines $o$ and $h$. At this point, the crystal structure changes orthorhombic to hexagonal. Owing to the transition, the following enthalpy change occurs, and an equal amount of heat quantity is consequently absorbed into the sample.

$$
\Delta H_{\mathrm{oh}}=H_{\mathrm{h}}-H_{\mathrm{o}}=T_{\mathrm{oh}}\left(S_{\mathrm{h}}-S_{\mathrm{o}}\right)
$$

where $H_{\mathrm{o}}$ and $H_{\mathrm{h}}$ are enthalpies of the orthorhombic and hexagonal phases, respectively. Peak 2 of the DSC curves in Figures 3 and 4 corresponds to this endothermic phenomenon. After the transfer at $T_{\text {oh }}$, the constrained sample proceeds along line $\mathrm{h}$, and then reaches $T_{\mathrm{hm}}$, the intersection of lines $\mathrm{h}$ and $\mathrm{m}$. At this point, the sample taking the hexagonal structure melts and changes to constrained, i.e., molecularly highly-oriented melt phase
Table III. Peak temperatures of constrained

\begin{tabular}{|c|c|c|c|c|c|}
\hline \multirow{2}{*}{ Sample } & Peak 1 & Peak 2 & Peak 3 & \multirow{2}{*}{$\begin{array}{l}\text { Method of } \\
\text { measurement }\end{array}$} & $\mathrm{HR}^{\mathrm{d}}$ \\
\hline & ${ }^{\circ} \mathrm{C}$ & ${ }^{\circ} \mathrm{C}$ & ${ }^{\circ} \mathrm{C}$ & & $\mathrm{K} \min ^{-1}$ \\
\hline Sample- $1^{\text {a }}$ & 143 & 155 & 159 & DSC & 5.0 \\
\hline Sample- $2^{\mathrm{a}}$ & 142 & 153 & 155 & DSC & 5.0 \\
\hline Sample- $3^{a}$ & 143 & 154 & 157 & DSC & 5.0 \\
\hline Sample- $4^{\mathrm{a}}$ & 143 & 153 & 158 & DSC & 5.0 \\
\hline Sample- $5^{b}$ & 144 & 153 & 164 & DSC & 5.0 \\
\hline Sample- $6^{c}$ & & 153 & 176 & DSC & 10.0 \\
\hline
\end{tabular}
UHMW-PE samples

a These data were obtained by the authors for samples wound round copper ring. ${ }^{b}$ A. J. Pennings and A. Zwijnenburg, J. Polym. Sci., Polym. Phys. Ed., 17, 1011 (1979). ${ }^{\text {c }}$ N. A. J. M. van Aerle and P. J. Lemstra, Polym. J., 20, 131 (1988). ${ }^{\mathrm{d}} \mathrm{HR}$ represents heating rate.

(line $\mathrm{m}$ ). Because of the melting, the tensile strength of the sample decreases considerably, so that its break and rapid contraction take place. Through this change, the sample downward transfers from line $\mathrm{m}$ to $\mathrm{m}^{\prime}$ of unconstrained melt phase at $T_{\mathrm{hm}}$ in the diagram. The melting of the hexagonal phase and the subsequent irreversible phenomena cause in total an enthalpy change

$$
\Delta H_{\mathrm{hm}^{\prime}}=H_{\mathrm{m}^{\prime}}-H_{\mathrm{h}}=H_{\mathrm{m}^{\prime}}-H_{\mathrm{m}}+T_{\mathrm{hm}}\left(S_{\mathrm{m}}-S_{\mathrm{h}}\right)
$$

where $H_{\mathrm{m}}$ and $H_{\mathrm{m}^{\prime}}$ are enthalpies of the constrained and unconstrained melt phases, respectively. A heat quantity to supply the required enthalpy change is absorbed into the sample and is responsible for peak 3 of DSC curves in Figures 3 and 4. After the characteristic transfer at $T_{\mathrm{hm}}$, the sample that is no longer constrained proceeds along line $\mathrm{m}^{\prime}$.

The melting process described on the basis of the proposed free energy diagram can explain the obtained experimental results successfully. For example, it explains that the hexagonal phase appears only under the constrained state competing with the thermal-contractile force. Furthermore, it does that the transition (peak 2) and melting (peak 3 ) of the constrained sample occur at respective constant temperatures as pointed out in Tables I and II because the intersection points of the diagram are constant under the identical condition for each sample. The break and rapid contraction succeeding the melting of the hexagonal phase are also explained. On the other hand, it should be noted that the melting process described on the basis of the diagram is an ideal one. In fact, there are unconstrained or insufficiently constrained portions that do not follow this process. These portions melt and transfer to line $\mathrm{m}^{\prime}$ of the unconstrained melt phase in the course of this ideal process.

Table III is a list of the peak temperatures measured by DSC for the constrained samples. The data of samples- 1 to 4 were measured by the authors, and those of samples-5 and -6 were referred from the literatures. ${ }^{7,8}$ Samples- 3 and -4 were prepared by drawing dry gel films made from as-polymerized UHMW-PE powder resins, "Hizex Million 340M" (Mitsui Petrochemical Industries Co., $\left.M_{\mathrm{v}}=c a .2 .7 \times 10^{6}\right)$ and a sample supplied by Showa Denko Co. $\left(M_{\mathrm{v}}=2.5 \times 10^{6}\right)$, respectively. They had an appearance of tape. The authors will mention the detailed preparation method in the following paper (Part II). As shown in the table, the temperature of peak 1 is almost constant, i.e., $142-144^{\circ} \mathrm{C}$, that is to say, the melting 
point of the unconstrained orthorhombic phase is not affected by the differences in the kinds of samples. The temperature of peak 2 , the transition point, is also within a very narrow temperature range, i.e., $153-155^{\circ} \mathrm{C}$. Besides these DSC data, there are X-ray data about this transition point. Based on them, the authors gave 155 and $153^{\circ} \mathrm{C}$ to constrained samples- 1 and -2 , respectively, and Pennings et al. gave $c a .150^{\circ} \mathrm{C}$ to their sample-5. These data are roughly close to those in the table. It should be noted that the DSC data of sample-6 in Table III and the X-ray data were obtained with respect to the samples constrained by embedding them in the epoxy resin, while the other DSC data were done for the samples constrained by winding them round the copper ring or wire. The fact that the transition points of the various samples constrained by the different methods are close to one another indicates that the temperature is hardly influenced by the differences in the kinds of samples and the constraining methods. Therefore, it is not expected to raise the transition point appreciably by changing the sample or constraining method in some degree. To raise it and improve the heat resistance of the constrained sample, it is probably necessary to change the sample state drastically by special methods such as the chemical cross-linkings. ${ }^{11,12}$ On the other hand, the temperature of peak 3 assigned to the melting of the hexagonal phase differs considerably according to the samples. Pennings et al. reported that constrained sample-5 used in their $\mathrm{X}$-ray measurement took the hexagonal phase even above $180^{\circ} \mathrm{C}$. As shown by these data, this temperature depends on the kind of sample and the constraining method remarkably. The crystals in the constrained fiber undergo the constraining stress that is equal to the thermalcontractile one at elevated temperatures. Because of the differences in molecular weight, preparation method, or constraining one, the stresses to the crystals in the different samples are expected to differ from one another. The state of the constrained melt phase is considerably influenced by the difference in the constraining stress, so that the energy line $m$ in Figure 11 also shifts by it. Such a factor may cause the differences in this peak temperature, i.e., the intersection point Thm, among the samples in the table. To clarify the well-defined origin, it is required to obtain more detailed information as to the melting process under the constrained state by studying about the effects of the constraining stress quantitatively.

Acknowledgements. The authors are grateful to Prof. M. Hikosaka of Hiroshima University for kindly discussions and very valuable advices about the free energy diagram and melting mechanism. This work was partly supported by a Grant-in-Aid for Scientific Research (No. 06750934) from the Ministry of Education, Science, and Culture of Japan and a grant from the Central Research Institute of Fukuoka University.

\section{REFERENCES}

1. P. Smith and P. J. Lemstra, J. Mater. Sci., 15, 505 (1980).

2. P. Smith and P. J. Lemstra, Polymer, 21, 1341 (1980).

3. P. Smith and P. J. Lemstra, and H. C. Booij, J. Polym. Sci., Polym. Phys. Ed., 19, 877 (1981).

4. K. Furuhata, T. Yokokawa, and K. Miyasaka, J. Polym. Sci., Polym. Phys. Ed., 22, 133 (1984).

5. H. Kyotani, S. Mitsuhashi, and M. Iguchi, Kobunshi Ronbunshu, 42, 849 (1985).

6. M. Matsuo, J. Soc. Rheology, Jpn., 13, 4 (1985)

7. A. J. Pennings and A. Zwijnenburg, J. Polym. Sci., Polym. Phys. Ed., 17, 1011 (1979).

8. N. A. J. M. van Aerle and P. J. Lemstra, Polym. J., 20, 131 (1988).

9. J. de Boer and A. J. Pennings, Polym. Bull., 5, 317 (1981).

10. J. de Boer and A. J. Pennings, Polym. Bull., 7, 309 (1982).

11. J. de Boer, H. J. van den Berg, and A. J. Pennings, Polymer, 25, 513 (1984).

12. M. Matsuo and C. Sawatari, Macromolecules, 19, 2028 (1986).

13. M. Yasuniwa, C. Nakafuku, and T. Takemura, Polym. J., 4, 526 (1973).

14. D. C. Bassett and B. Turner, Nature (Phys. Sci.), 240, 146(1972).

15. D. C. Bassett, S. Block, and C. J. Piermarini, J. Appl. Phys., 45, 4146 (1974).

16. M. Yasuniwa, R. Enoshita, and T. Takemura, Jpn. J. Appl. Phys., 15, 1421 (1976)

17. H. Tanaka and T. Takemura, Polym. J., 12, 355 (1980).

18. T. Yamamoto, H. Miyaji, and K. Asai, Jpn. J. Appl. Phys., 16, 1891 (1977).

19. S. Tsubakihara, A. Nakamura, and M. Yasuniwa, Polym. J., 23, 1317 (1991). 